\title{
Randomized trial of intravenous iron-induced hypophosphatemia
}

\author{
Myles Wolf, ${ }^{1,2}$ Glenn M. Chertow, ${ }^{3,4}$ Iain C. Macdougall,, Robert Kaper, ${ }^{6}$ Julie Krop, ${ }^{6}$ \\ and William Strauss ${ }^{6}$ \\ 'Division of Nephrology, Department of Medicine, and ²Duke Clinical Research Institute, Duke University School of \\ Medicine, Durham, North Carolina, USA. ${ }^{3}$ Division of Nephrology, Department of Medicine and ${ }^{4}$ Department of Health \\ Research and Policy, Stanford University, Stanford, California, USA. ${ }^{5}$ Renal Unit, King's College Hospital, London, United \\ Kingdom. ${ }^{6}$ AMAG Pharmaceuticals, Inc., Waltham, Massachusetts, USA.
}

BACKGROUND. Hypophosphatemia can complicate intravenous iron therapy, but no head-to-head trials compared the effects of newer intravenous iron formulations on risks and mediators of hypophosphatemia.

METHODS. In a randomized, double-blinded, controlled trial of adults with iron deficiency anemia from February 2016 to January 2017, we compared rates of hypophosphatemia in response to a single FDA-approved course of ferric carboxymaltose $(n=1,000)$ or ferumoxytol $(n=997)$. To investigate pathophysiological mediators of intravenous iron-induced hypophosphatemia, we nested within the parent trial a physiological substudy (ferric carboxymaltose, $n=98$; ferumoxytol, $n=87$ ) in which we measured fibroblast growth factor 23 (FGF23), calcitriol, and parathyroid hormone (PTH) at baseline and 1, 2, and 5 weeks later.

RESULTS. The incidence of hypophosphatemia was significantly higher in the ferric carboxymaltose versus the ferumoxytol group ( $<2.0 \mathrm{mg} / \mathrm{dl}, 50.8 \%$ vs. $0.9 \% ;<1.3 \mathrm{mg} / \mathrm{dl}, 10.0 \%$ vs. $0.0 \% ; P<$ 0.001 ), and hypophosphatemia persisted through the end of the 5 -week study period in $29.1 \%$ of ferric carboxymaltose-treated patients versus none of the ferumoxytol-treated patients $(P<0.001)$. Ferric carboxymaltose, but not ferumoxytol, increased circulating concentrations of biologically active FGF23 (mean within-patient percentage change from baseline to week 2 peak: $+302.8 \pm 326.2 \%$ vs. $+10.1 \pm 61.0 \% ; P<0.001$ ), which was significantly associated with contemporaneous hypophosphatemia, renal phosphate wasting, and decreased serum calcitriol and calcium, and increased PTH concentrations.

Conflict of interest: MW serves as a consultant to AMAG Pharmaceuticals Inc. and Luitpold Pharmaceuticals Inc. GMC serves as a consultant to AMAG Pharmaceuticals Inc. ICM received consultancy fees and honoraria from AMAG Pharmaceuticals Inc. and Vifor Pharma. At the time of the study, RK held equity in and was a full-time employee of AMAG Pharmaceuticals Inc. JK and WS hold equity in and are full-time employees of AMAG Pharmaceuticals Inc.

License: Copyright 2018, American Society for Clinical Investigation.

Submitted: August 27, 2018 Accepted: October 31, 2018 Published: December 6, 2018

Reference information: JCI Insight. 2018;3(23):e124486. https://doi.org/10.1172/jci. insight.124486.
CONCLUSIONS. Ferric carboxymaltose rapidly increases biologically active FGF23 in patients with iron deficiency anemia. Paralleling hereditary and other acquired syndromes of hypophosphatemic rickets/osteomalacia, ferric carboxymaltose-induced FGF23 elevation triggers a pathophysiological cascade of renal phosphate wasting, calcitriol deficiency, and secondary hyperparathyroidism that frequently culminates in hypophosphatemia.

TRIAL REGISTRATION. ClinicalTrials.gov, NCT02694978

FUNDING. AMAG Pharmaceuticals, Inc.

Role of the funding source: This study was supported by AMAG Pharmaceuticals, Inc. The academic investigators designed the clinical trial, performed the analyses, and authored the manuscript with input from the coauthors from AMAG Pharmaceuticals, Inc.

\section{Introduction}

Iron deficiency anemia is a global health problem that is most commonly caused by excessive blood loss, impaired intestinal iron absorption, or chronic inflammation $(1,2)$. Oral iron is the first-line treatment for most patients with iron deficiency, but insufficient gastrointestinal absorption and frequent adverse effects limit efficacy and adherence, requiring the provision of intravenous iron (1). While multiple intravenous iron formulations have been in use for decades, newer formulations, such as ferric carboxymaltose and 
ferumoxytol, were specifically developed to maximize convenience for outpatient use by enabling safe and more rapid administration of higher doses of iron than older formulations $(1,2)$.

Coincident with increased utilization of intravenous iron, reports are accumulating of patients who developed severe hypophosphatemia with serious musculoskeletal complications following their administration, including osteomalacia, fragility fractures, and hypoxemia (3-12). Although the molecular mechanism of hypophosphatemia is not fully understood, certain forms of intravenous iron may increase circulating concentrations of fibroblast growth factor 23 (FGF23) (13-16). Constitutively elevated serum FGF23 concentrations in rare prototypical diseases of primary FGF23 excess, including hereditary hypophosphatemic rickets and tumor-induced osteomalacia, cause hypophosphatemia and bone demineralization by stimulating renal phosphate wasting and suppressing serum concentrations of 1,25-dihydroxyvitamin D (14, 15, 17-20).

Retrospective and small pilot studies suggest discrepant rates of hypophosphatemia in response to different forms of intravenous iron $(13,15,21-23)$, but until recently, no randomized trials directly compared two of the newer formulations. The FIRM trial (IDA-304) was a randomized, multicenter, double-blinded, controlled, noninferiority trial that compared the safety and efficacy of a single US FDA-approved course of intravenous ferumoxytol versus ferric carboxymaltose for treatment of iron deficiency anemia (24). In the primary analyses, ferumoxytol was noninferior to ferric carboxymaltose for hypersensitivity reactions, including anaphylaxis and hypotension, and for raising hemoglobin (24). Here, we report results of prespecified secondary analyses of the incidence of, and clinical risk factors for, hypophosphatemia in the parent trial and its nested physiological substudy. We tested the hypotheses that the incidence and severity of hypophosphatemia are higher in response to ferric carboxymaltose versus ferumoxytol; that renal phosphate wasting due to increases in biologically active FGF23 concentrations underlies hypophosphatemia; and that the etiology of iron deficiency influences risk of intravenous iron-induced hypophosphatemia.

\section{Results}

The FIRM trial enrolled 1,997 patients aged $\geq 18$ years with iron deficiency anemia, defined as hemoglobin $<12.0 \mathrm{~g} / \mathrm{dl}$ for women and $<14.0 \mathrm{~g} / \mathrm{dl}$ for men, and transferrin saturation (TSAT) $\leq 20 \%$ or ferritin $\leq 100 \mathrm{ng} / \mathrm{ml}$, who previously did not tolerate or insufficiently responded to oral iron; pregnant women and patients being treated with dialysis were excluded. The study recruited patients in the US, Canada, and Europe from February 2016 to January 2017. All patients and site investigators were blinded to the assignment of the intervention. Within the parent FIRM trial, we nested a physiological substudy in which we performed repeated measurements of phosphate homeostasis in blood and urine at baseline (week 0 ) and at weeks 1, 2, and 5 after baseline. We offered enrollment in the physiological substudy to consecutive participants at US-based sites until the full sample of at least 180 patients was reached.

We randomized patients who provided written informed consent 1:1 to receive $510 \mathrm{mg}$ ferumoxytol (Feraheme; AMAG Pharmaceuticals, Inc.; parent trial, $n=997$; substudy, $n=87$ ) or 750 mg ferric carboxymaltose (Injectafer; American Regent, Inc.; parent trial, $n=1,000$; substudy, $n=98$ ), administered intravenously over $\geq 15$ minutes at baseline and 1 week later. The total dose of elemental iron was 1,020 mg in the ferumoxytol arm and 1,500 $\mathrm{mg}$ in the ferric carboxymaltose arm. The CONSORT diagram for the parent trial and study schema of the nested physiological substudy are presented in Figure 1.

Baseline characteristics of the parent trial and the nested physiological substudy populations were similar across treatment groups (Table 1). With the exception of modest differences in chronic kidney disease (CKD) prevalence and estimated glomerular filtration rate (eGFR), characteristics of patients in the parent trial and the nested physiological substudy were qualitatively similar.

Parent trial. The incidence of severe hypophosphatemia $(<2.0 \mathrm{mg} / \mathrm{dl})$ and extreme hypophosphatemia $(<1.3 \mathrm{mg} / \mathrm{dl})$ was significantly higher in the ferric carboxymaltose group versus the ferumoxytol group ( $<2.0 \mathrm{mg} / \mathrm{dl}, 50.8 \%$ vs. $0.9 \% ;<1.3 \mathrm{mg} / \mathrm{dl}, 10.0 \%$ vs. $0.0 \% ; P<0.001)$. In the ferric carboxymaltose group, risk of hypophosphatemia was magnified among patients without $\mathrm{CKD}$ but was also observed among patients with CKD ( $<2.0 \mathrm{mg} / \mathrm{dl}, 64.0 \%$ vs. $21.5 \% ;<1.3 \mathrm{mg} / \mathrm{dl}, 13.7 \%$ vs. $1.9 \% ; P<0.001)$. Rates of hypophosphatemia at each study visit are shown in Figure 2. Compared with none of the ferumoxytol-treated patients, $29.1 \%$ of ferric carboxymaltose-treated patients remained hypophosphatemic at the end of the 5-week study period $(P<0.001)$, including $4.7 \%$ with extreme hypophosphatemia $<1.3 \mathrm{mg} / \mathrm{d} 1$.

As reported previously (24), mean serum phosphate remained stable in the ferumoxytol group, but decreased significantly to its nadir at week 2 in the ferric carboxymaltose group (mean difference between 
A

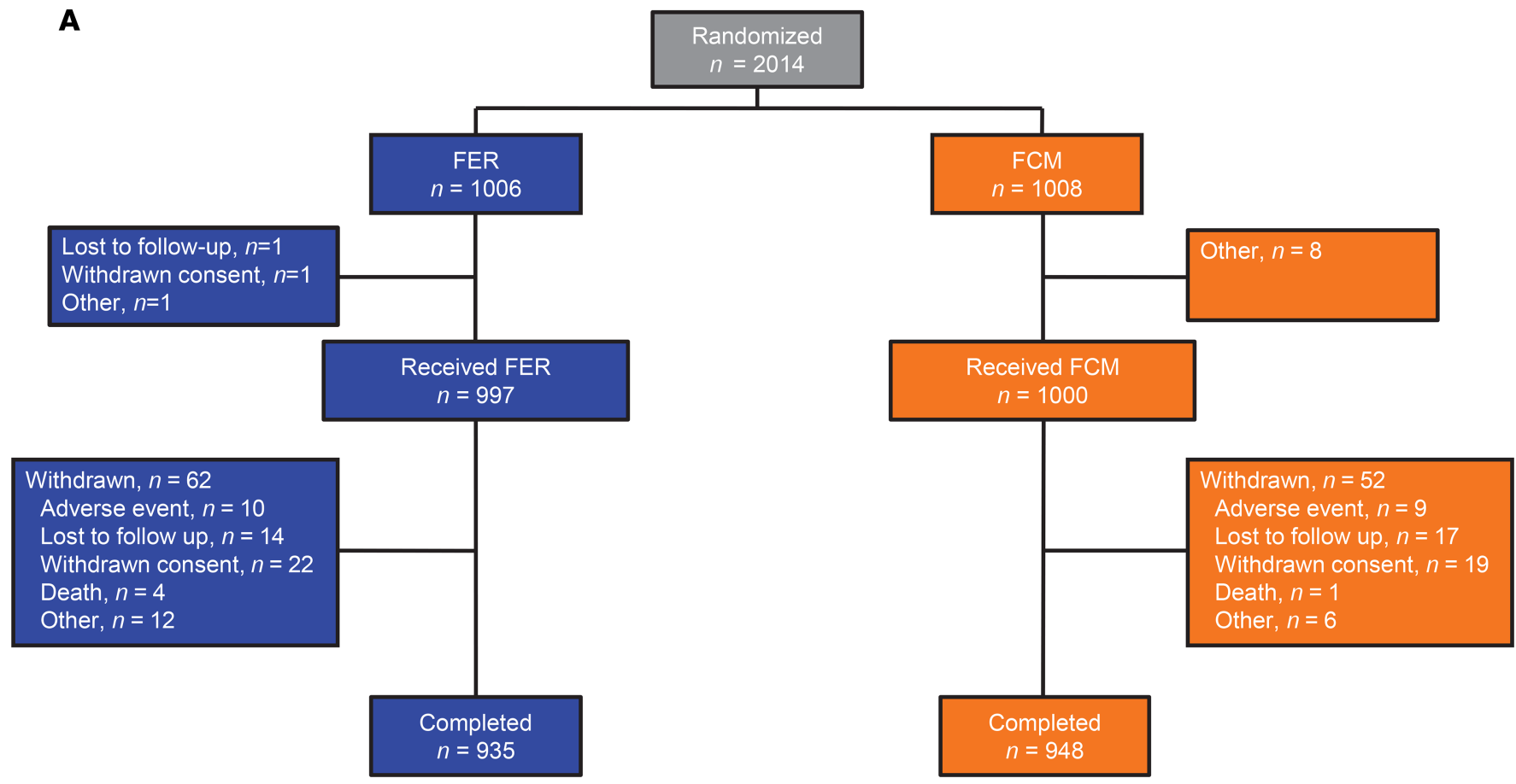

B

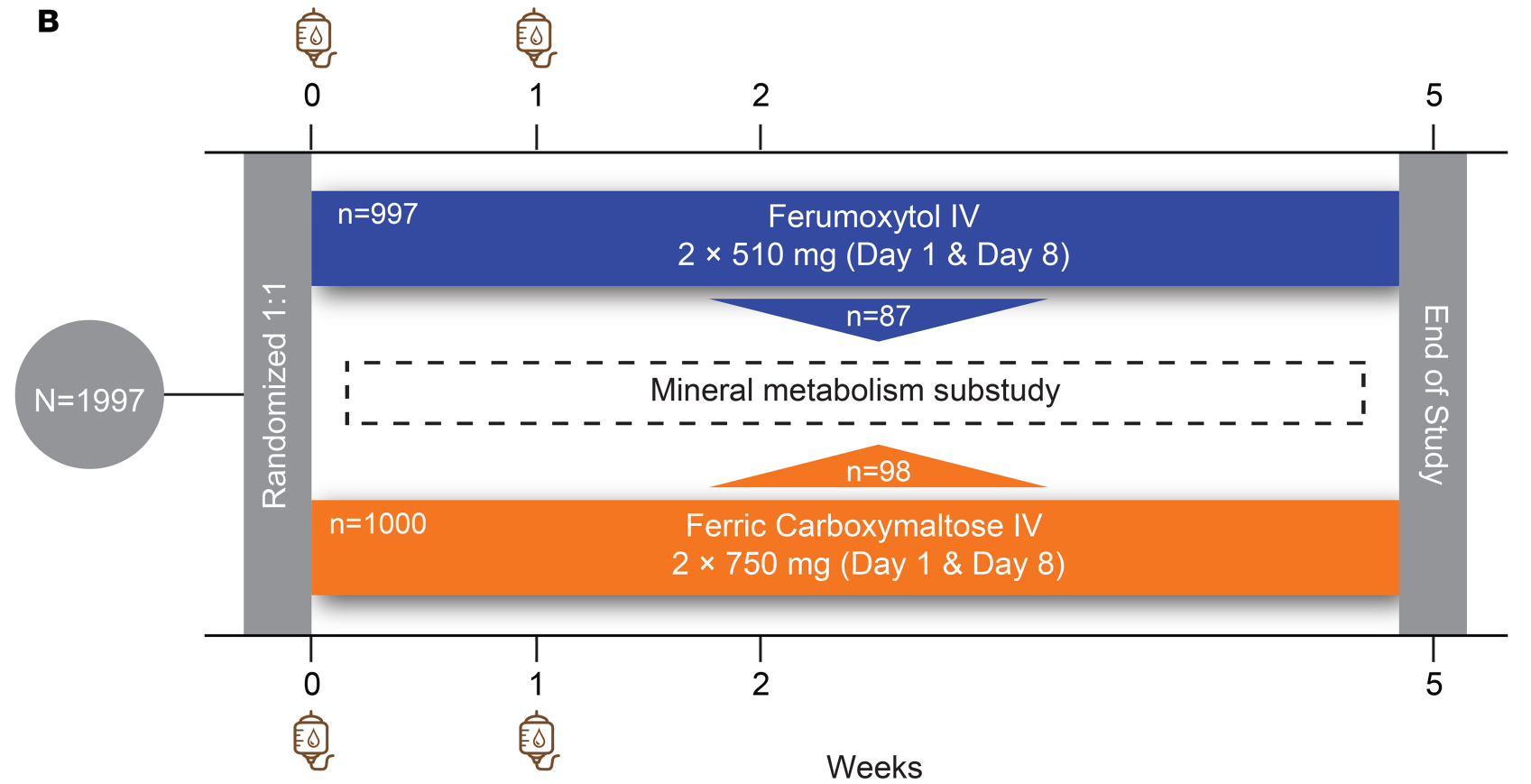

Figure 1. The FIRM trial and its nested physiological substudy. (A) CONSORT diagram of the FIRM trial. (B) After providing written informed consent, 1,997 patients were randomized to receive intravenous (IV) ferumoxytol $(n=997)$ or ferric carboxymaltose $(n=1,000)$ on day 0 and 1 week later. Additional study visits occurred at weeks 2 and 5 after enrollment. A subgroup of US-based patients enrolled in the FIRM trial's nested physiological substudy (ferumoxytol, $n=87$; ferric carboxymaltose, $n=98$ ) and underwent more detailed laboratory testing of phosphate homeostasis.

groups in change from baseline to week $2,1.51 \mathrm{mg} / \mathrm{dl} ; 95 \%$ confidence interval [95\% $\mathrm{CI}] 1.45-1.57 ; P<0.001$ ). The decrease in serum phosphate in the ferric carboxymaltose group was associated with a concomitant increase in urinary fractional excretion of phosphate $(\mathrm{FEPi})$ that did not occur in the ferumoxytol group (mean difference between groups in change in FEPi, 9.2\%; 95\%CI 8.2-10.2; $P<0.001$; overall correlation between change from baseline to week 2 in serum phosphate and FEPi in all patients, $\rho=-0.43 ; P<0.001$ ). The differential effects of the iron formulations on serum phosphate were magnified among patients without CKD but 
Table 1. Baseline characteristics of the study populations of the parent trial and nested physiological substudy according to randomized iron treatment group

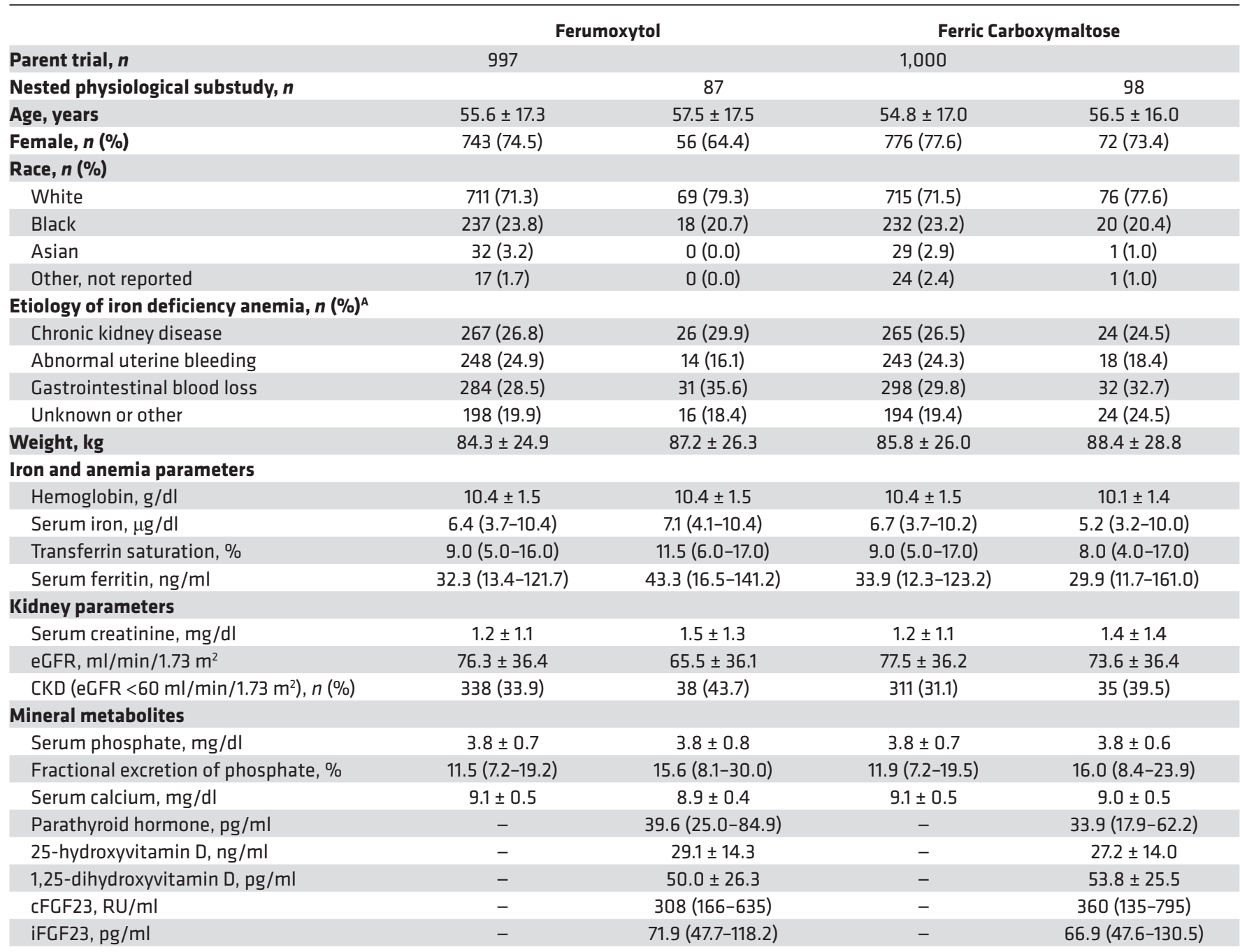

Results are reported as proportions, mean \pm standard deviation or median (interquartile ranges [25th-75th percentiles]). ${ }^{A}$ As reported by site investigators. CFGF23, C-terminal fibroblast growth factor 23; CKD, chronic kidney disease; eGFR, estimated glomerular filtration rate; iFCF23, intact fibroblast growth factor 23.

were also detectable among those with $\mathrm{CKD}$ (mean difference between groups in change from baseline to week $2,1.73 \mathrm{mg} / \mathrm{dl}, 95 \%$ CI $1.66-1.80$ vs. $1.02 \mathrm{mg} / \mathrm{dl}, 95 \%$ CI $0.90-1.14)$.

Clinical risk factors for hypophosphatemia. In a multivariable model of the parent trial that considered age, sex, race, body weight, etiology of iron deficiency anemia, and baseline phosphate, hemoglobin, iron, ferritin, and eGFR, treatment with ferric carboxymaltose versus ferumoxytol was the strongest risk factor for incident hypophosphatemia (odds ratio $250.6,95 \%$ CI $115.4-544.5$; Table 2). Other factors independently associated with increased risk of hypophosphatemia included higher eGFR and hemoglobin, lower body weight and serum phosphate, and abnormal uterine bleeding as the etiology of iron deficiency anemia; CKD as the etiology of iron deficiency anemia was associated with lower risk. In analyses of risk of persistent hypophosphatemia at study end, the effects of lower body weight and serum phosphate strengthened, and Black race emerged as an additional independent risk factor (Table 2). The results were qualitatively similar when restricted to patients without CKD (data not shown), except that lower baseline ferritin was also independently associated with increased risk of hypophosphatemia (odds ratio 0.68 per $100 \mathrm{mg} / \mathrm{dl}$ increase, $95 \% \mathrm{CI} 0.52-0.90$ ).

Nested physiological substudy. In the nested physiological substudy, we repeatedly measured phosphate, calcium, vitamin D, and parathyroid hormone (PTH) using standard clinical assays, and FGF23 using two complementary research assays. The C-terminal FGF23 assay (cFGF23) detects full-length biologically 


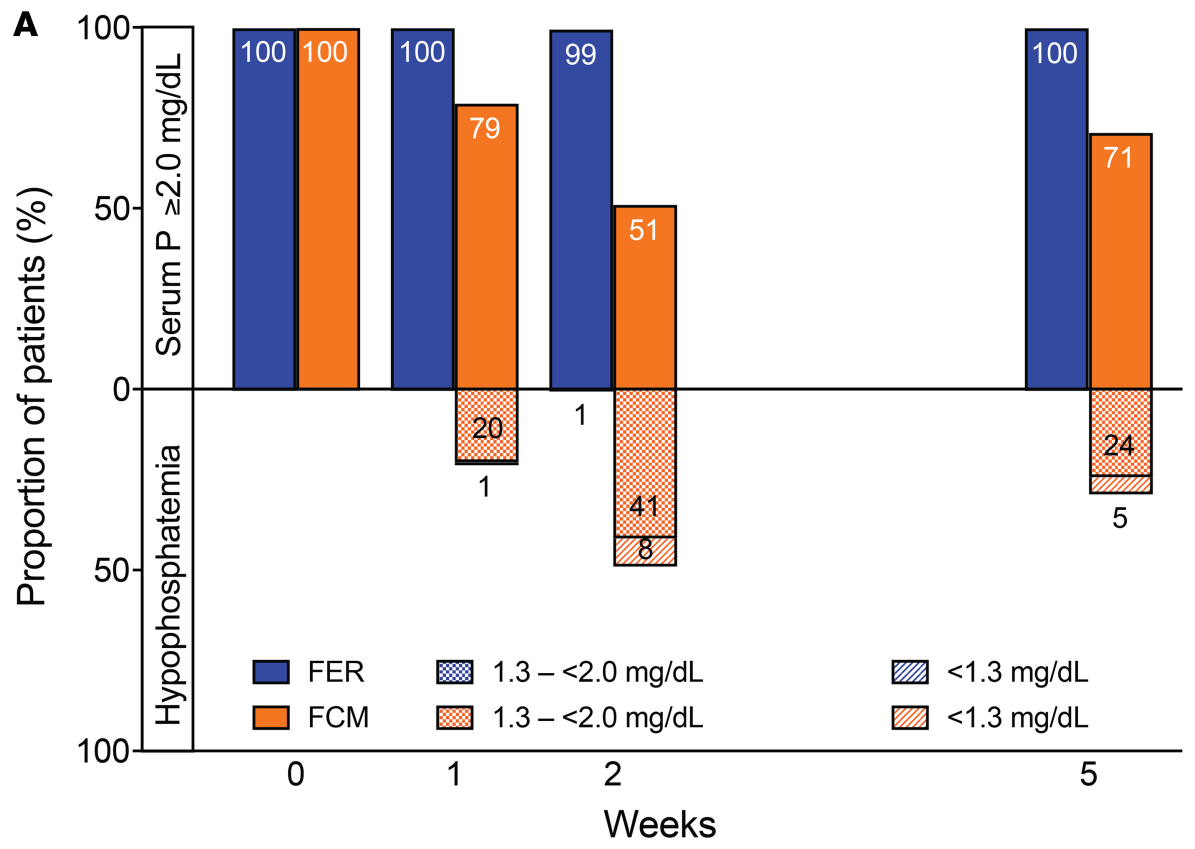

Figure 2. Hypophosphatemia in the parent FIRM trial. (A) Proportions of patients with serum phosphate $(P) \geq 2.0 \mathrm{mg} / \mathrm{dl}$, severe hypophosphatemia $<2.0$ or extreme hypophosphatemia $<1.3 \mathrm{mg} / \mathrm{dl}$ in the ferumoxytol (FER; $n=997$ ) and ferric carboxymaltose (FCM; $n=1,000$ ) treatment groups at each study visit. (B) Proportions of FCM-treated patients with serum $P \geq 2.0 \mathrm{mg} / \mathrm{dl}$, severe hypophosphatemia $<2.0$ or extreme hypophosphatemia $<1.3 \mathrm{mg} / \mathrm{dl}$ at each study visit, stratified by presence $(n=311)$ or absence $(n=689)$ of chronic kidney disease (CKD). Intravenous iron was administered at baseline (week 0 ) and at week 1 after blood and urine were collected.

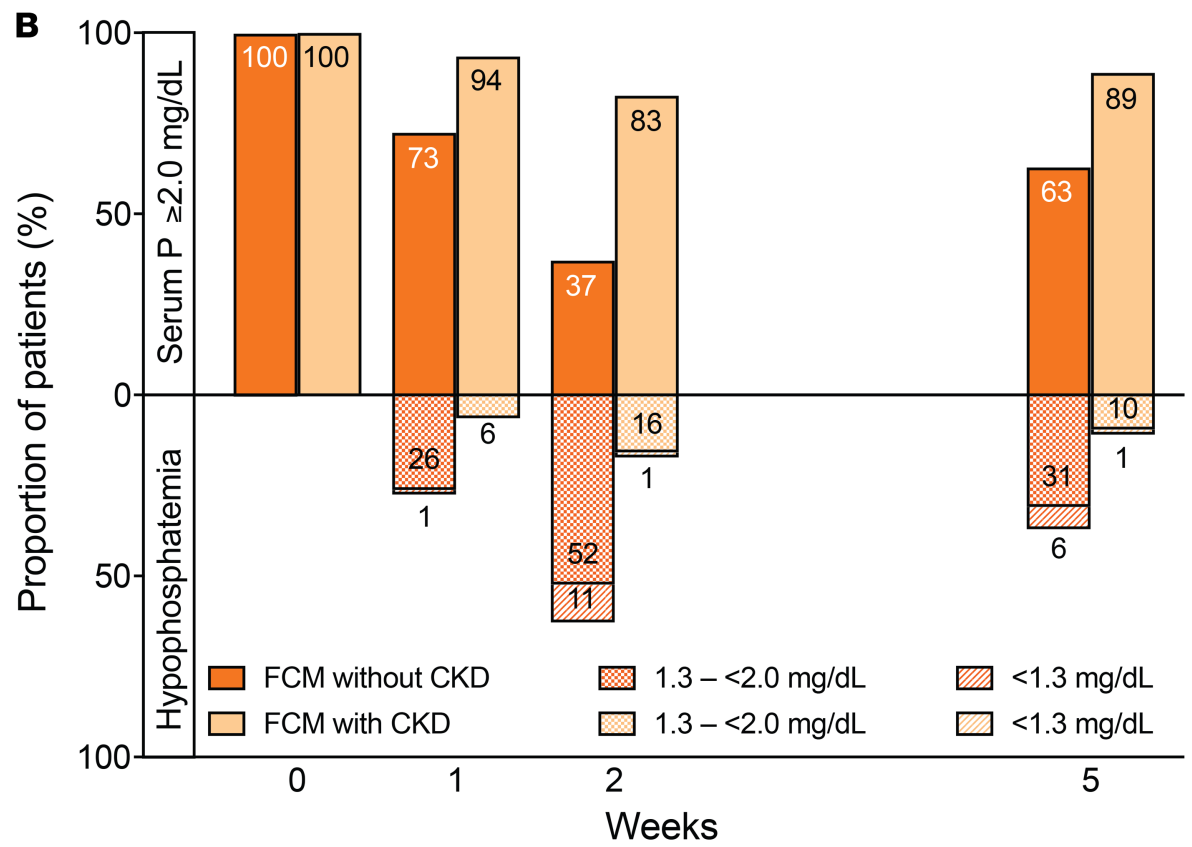

active FGF23 and its C-terminal fragments, which do not affect phosphate homeostasis; the intact FGF23 assay (iFGF23) exclusively detects full-length, biologically active FGF23 (17).

At baseline, serum cFGF23 concentrations were similarly elevated in the ferric carboxymaltose and ferumoxytol groups, whereas iFGF23 levels and thus, serum phosphate, were within the population reference range in both groups (Table 1). Although patients with versus without CKD had higher baseline serum iFGF23 concentrations (median [interquartile range, IQR] 121 [73-269] vs. 55 [44-77] pg/ml; $P<$ 0.001 ), there was no significant difference in baseline plasma cFGF23 among patients with versus without CKD (median [IQR] 348 [181-502] vs. 350 [133-845] RU/ml; $P=0.76$ ). At baseline, lower serum ferritin and hemoglobin were independently associated with higher cFGF23, whereas lower eGFR and higher serum phosphate were independently associated with higher iFGF23.

The ferumoxytol group maintained stable serum phosphate and urinary phosphate excretion throughout the trial (Figure 3, A and B). In the ferric carboxymaltose group, serum phosphate progressively decreased from baseline to week 1 before reaching its nadir at week 2 (mean difference between iron 
Table 2. Baseline clinical risk factors for incident hypophosphatemia at any time during the trial and for persistent hypophosphatemia at study end in the overall parent trial population

\begin{tabular}{|c|c|c|c|c|}
\hline \multirow[b]{2}{*}{ Risk factor } & \multicolumn{2}{|c|}{ Incident hypophosphatemia } & \multicolumn{2}{|c|}{ Persistent hypophosphatemia } \\
\hline & Odds ratio & $95 \% \mathrm{Cl}$ & Odds ratio & $95 \% \mathrm{Cl}$ \\
\hline FCM vs. FER & 250.6 & $115.4-544.5$ & 271.4 & $66.5-1,106.7$ \\
\hline eGFR, per $10 \mathrm{ml} / \mathrm{min} / 1.73 \mathrm{~m}^{2}$ increase & 1.07 & $1.01-1.13$ & - & - \\
\hline Hemoglobin, per $1 \mathrm{~g} / \mathrm{dl}$ increase & 1.24 & $1.12-1.38$ & 1.30 & $1.16-1.46$ \\
\hline Weight, per $10 \mathrm{~kg}$ increase & 0.92 & $0.87-0.97$ & 0.79 & $0.73-0.86$ \\
\hline Serum phosphate, per $1 \mathrm{mg} / \mathrm{dl}$ increase & 0.31 & $0.23-0.41$ & 0.24 & $0.17-0.34$ \\
\hline \multicolumn{5}{|l|}{ Etiology of iron deficiency anemia } \\
\hline Uterine bleeding vs other/unknown & 1.81 & $1.18-2.76$ & - & - \\
\hline CKDA vs. other/unknown & 0.38 & $0.22-0.64$ & 0.35 & $0.20-0.62$ \\
\hline Black vs. White race & - & - & 1.87 & $1.26-2.79$ \\
\hline \multicolumn{5}{|c|}{$\begin{array}{l}\text { Results are from final parsimonious multivariable models that initially considered the following baseline variables as candidate risk factors: age, sex, race, } \\
\text { body weight, etiology of iron deficiency anemia, serum phosphate, hemoglobin, iron, ferritin, eGFR, and randomized treatment with ferric carboxymaltose } \\
\text { or ferumoxytol. A As reported by site investigators. CI, confidence interval; CKD, chronic kidney disease; eGFR, estimated glomerular filtration rate; FCM, } \\
\text { ferric carboxymaltose; FER, ferumoxytol. }\end{array}$} \\
\hline
\end{tabular}

treatment groups in change from baseline to week $2,1.5 \mathrm{mg} / \mathrm{dl} ; 95 \% \mathrm{CI} 1.3-1.7 ; P<0.001$ ), while FEPi progressively increased (mean difference between iron treatment groups in change from baseline to week 2, 7.3\%; 95\%CI 2.3-12.3; $P=0.004$; Figure 3, A and B). Simultaneously, serum iFGF23 approximately doubled by week 1 and then doubled again by week 2 in the ferric carboxymaltose group, while remaining unchanged in the ferumoxytol group (Figure 3C and Table 3). Plasma cFGF23 decreased significantly in both groups (Figure 3D and Table 3). Compared with ferumoxytol, ferric carboxymaltose induced significant decreases in serum 1,25-dihydroxyvitamin $\mathrm{D}$ (mean within-patient percentage change from baseline to week $2,-60.4 \pm 25.9 \%$ vs. $-2.5 \pm 28.0 \% ; P<0.001$; Figure $3 \mathrm{E}$ ) and calcium (mean difference between iron treatment groups in change from baseline to week $2,0.37 \mathrm{mg} / \mathrm{dl} ; 95 \% \mathrm{CI} 0.25-0.50 ; P<0.001$; Figure $3 \mathrm{~F}$ ), and significant increases in PTH that peaked at week 5 (Figure $3 \mathrm{G}$ and Table 3). Serum 25-hydroxyvitamin $\mathrm{D}$ did not change throughout the study in either group (Figure $3 \mathrm{H}$ ). All results were qualitatively similar when restricted to patients without CKD (Figure 3 and Table 3).

Reductions in serum phosphate from baseline to week 2 and baseline to week 5 were associated with contemporaneous increases in iFGF23, FEPi, and PTH, and decreases in 1,25-dihydroxyvitamin D and calcium, but not with changes in cFGF23 (Table 4). These results were similar when restricted to patients without CKD except that the associations between change in serum phosphate and changes in iFGF23, 1,25-dihydroxyvitamin D, and PTH strengthened (Table 4).

\section{Discussion}

In this randomized trial of patients with iron deficiency anemia of diverse causes, treatment with ferric carboxymaltose induced hypophosphatemia in more than half of all patients and nearly two-thirds of those with normal kidney function compared with fewer than $1 \%$ of those who received ferumoxytol. Hypophosphatemia was detectable within 1 week of ferric carboxymaltose administration, peaked in frequency by week 2 , and persisted through the end of the 5-week study in nearly one-third of patients. The nadir in serum phosphate at week 2 coincided with an approximate 4-fold mean increase in circulating, full-length, biologically active FGF23 that resulted in renal phosphate wasting, decreased serum 1,25-dihydroxyvitamin D and calcium, and increased PTH concentrations. Based on these results and previously published pilot studies (14, 15), we conclude that ferric carboxymaltose rapidly increases biologically active FGF23 and recapitulates in just weeks the pathophysiological cascade that unfolds over months to years in other disorders of primary FGF23 excess, such as hereditary hypophosphatemic rickets and tumor-induced osteomalacia $(19,20)$.

Iron deficiency stimulates FGF23 transcription but does not cause hypophosphatemia because increased FGF23 production is coupled to increased FGF23 cleavage within osteocytes, which maintains normal circulating concentrations of full-length, biologically active hormone (17, 25). The parallel increases in FGF23 production and cleavage in untreated iron deficiency results in secretion of large 


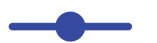

FMX (all participants)
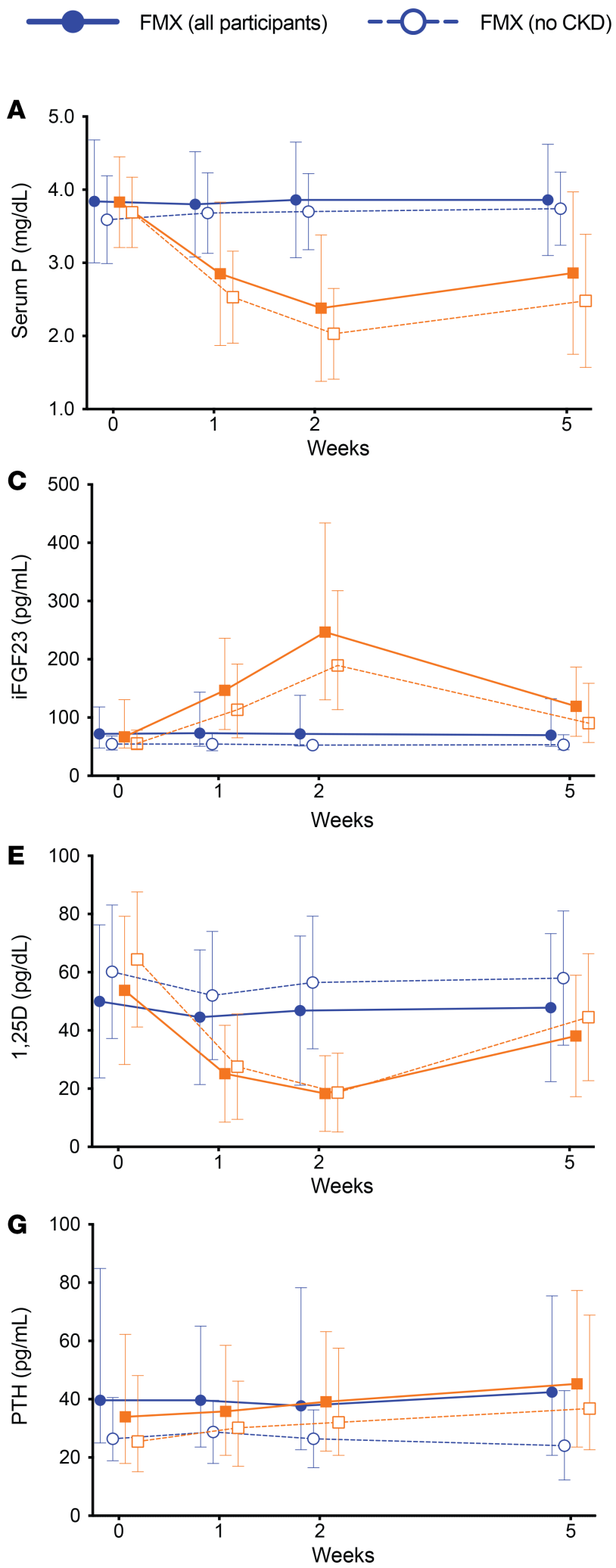
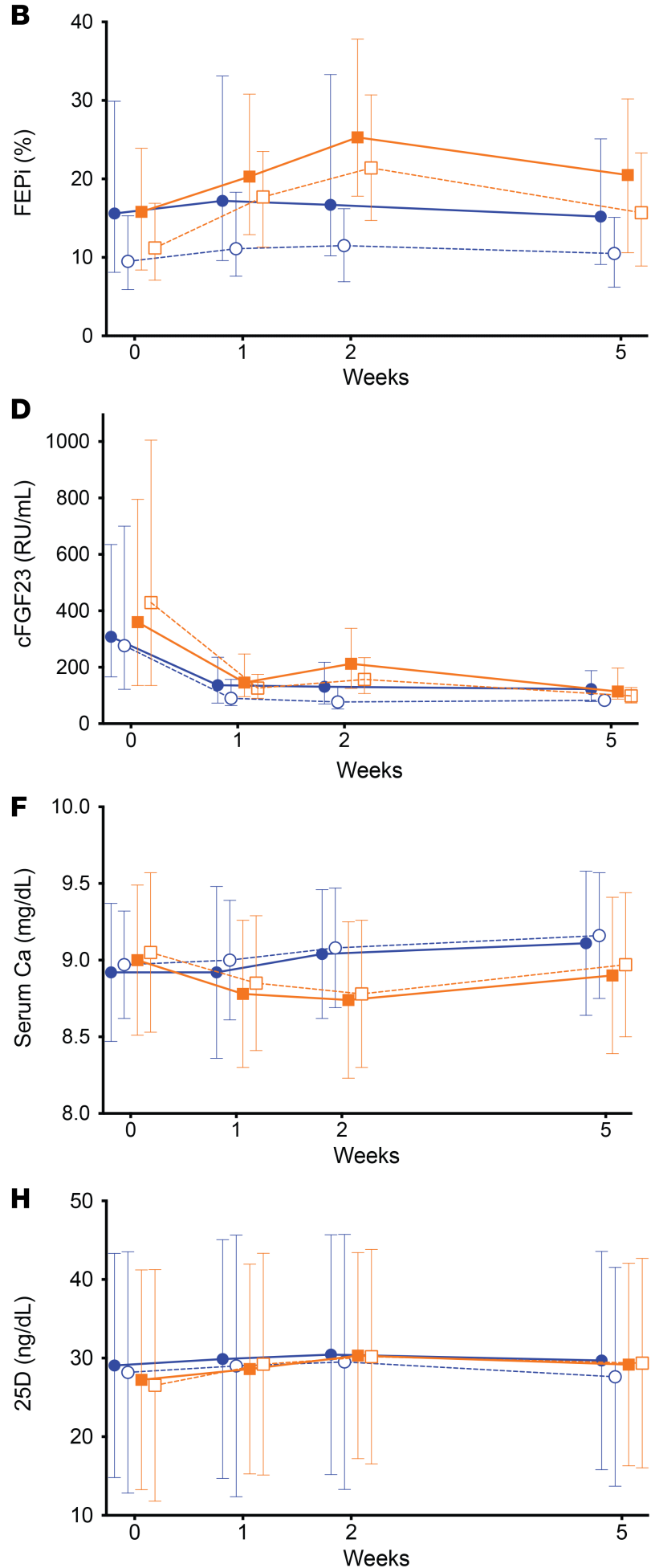

Figure 3. Phosphate homeostatic parameters in the nested physiological substudy. Changes over time in all patients in the ferumoxytol (FER; $n$ $=87$ ) and ferric carboxymaltose (FCM; $n=98)$ treatment groups (solid lines) and in the subgroups of patients without chronic kidney disease (CKD; FER, $n=49 ; \mathrm{FCM}, n=63$; dashed lines) in levels of (A) mean serum phosphate (P); (B) median fractional excretion of phosphate (FEPi); (C) median intact fibroblast growth factor 23 (iFGF23); (D) median C-terminal fibroblast growth factor 23 (cFGF23); (E) mean 1,25-dihydroxyvitamin D (1,25D); (F) mean serum calcium (Ca); (G) median parathyroid hormone (PTH); and (H) mean 25-hydroxyvitamin D (25D). Error bars are standard deviations (SDs) for means, and interquartile ranges (25th-75th percentiles) for medians. Intravenous iron was administered at baseline (week 0 ) and at week 1 after blood and urine were collected. 
Table 3. Percentage changes in phosphaturic hormones from baseline to each subsequent visit in the entire nested physiological substudy and within its subgroup without CKD

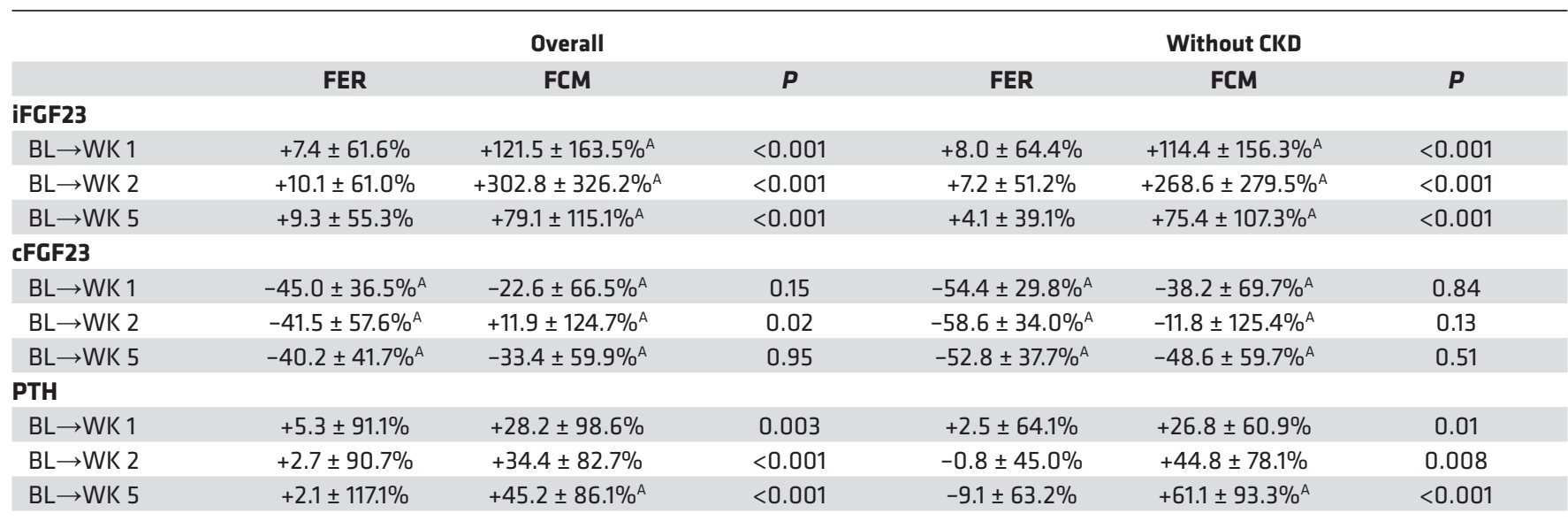

Results are reported as mean \pm standard deviation. $P$ values in columns correspond to comparisons between the iron treatment groups. ${ }^{A}$ Connotes $P<$ 0.001 for within-group comparisons ( $P$ value adjusted for the 36 tests). BL, baseline; CFGF23, C-terminal fibroblast growth factor 23; FER, ferumoxytol; FCM, ferric carboxymaltose; iFGF23, intact fibroblast growth factor 23; PTH, parathyroid hormone; WK, week.

amounts of C-terminal fragments into circulation that register as high cFGF23 but normal iFGF23 concentrations (17). Consistent with this mechanism, we demonstrated elevated cFGF23 at baseline, the magnitude of which related only to the severity of iron deficiency, whereas iFGF23 concentrations were normal, except among patients with CKD, in whom iFGF23 is known to rise in response to impaired kidney function (26). Following ferumoxytol treatment, repletion of iron stores reduced the stimulus for FGF23 transcription and thus the requisite amount of FGF23 cleavage, which was reflected in the rapid reduction in plasma cFGF23 but persistently normal serum iFGF23 concentrations that we observed. In contrast, ferric carboxymaltose increased iFGF23 at the same time that cFGF23 was decreasing (this group's week 2 uptick in CFGF23 reflects the assay's capture of C-terminal fragments and full-length hormone). Although the mechanism for this phenomenon is unknown, these data argue against a class effect of intravenous iron and suggest that ferric carboxymaltose uncouples the balance between FGF23 production and cleavage, perhaps by inhibiting FGF23 cleavage in osteocytes (14), or by activating FGF23 production in ectopic sites that do not express the FGF23 cleavage apparatus. Future studies that elucidate mechanisms of the synchronized regulation of FGF23 transcription and cleavage will not only help to explain why certain intravenous iron preparations raise serum iFGF23, but may also have important therapeutic applications to other pathological states of excessive FGF23 cleavage, such as tumoral calcinosis, or reduced FGF23 cleavage, such as CKD (27-29).

This study offers new insights into disordered phosphate homeostasis in CKD. Phosphate is filtered by the glomerulus and reabsorbed only in the proximal tubule; phosphate that escapes proximal reabsorption due to inhibition of sodium-phosphate cotransporters by FGF23 or PTH is excreted in the urine (30). Since the amount of phosphate that is filtered decreases as eGFR declines, it is not surprising that a history of $\mathrm{CKD}$ and lower eGFR attenuated risk of hypophosphatemia in this study. However, our observation that reduced serum phosphate and overt hypophosphatemia were detectable in a substantial number of ferric carboxymaltose-treated patients with $\mathrm{CKD}$ indicates that basal phosphate reabsorption is not maximally suppressed in CKD, despite high serum FGF23 and PTH concentrations. Instead, further increases in FGF23 induced by ferric carboxymaltose can further reduce phosphate reabsorption and further suppress 1,25-dihydroxyvitamin D concentrations. These data argue against tubular resistance to FGF23 via reduced expression of its renal coreceptor, klotho, as a primary cause of secondary FGF23 excess in CKD, and reinforce the need for additional investigation into mechanisms of FGF23 elevation in CKD (31).

The overall results of the current randomized trial align with those from an earlier pilot trial of otherwise healthy young women with iron deficiency due to heavy uterine bleeding who were randomized to receive up to $1,000 \mathrm{mg}$ of ferric carboxymaltose or iron dextran in a single dose (15). By 1 day later, 
Table 4. Spearman's correlations $(\rho)$ and tests of significance $(P)$ of contemporaneous changes in serum phosphate and changes in other parameters of phosphate homeostasis from baseline to week $\mathbf{2}$ and from baseline to week $\mathbf{5}$ in the entire nested physiological substudy and within its subgroup without CKD

\begin{tabular}{|c|c|c|c|c|c|c|c|c|}
\hline & \multicolumn{4}{|c|}{ From baseline to week 2} & \multicolumn{4}{|c|}{ From baseline to week 5} \\
\hline & \multicolumn{2}{|c|}{ Overall $n=185$} & \multicolumn{2}{|c|}{ Without CKD $n=112$} & \multicolumn{2}{|c|}{ Overall $n=185$} & \multicolumn{2}{|c|}{ Without CKD $n=112$} \\
\hline & $\rho$ & $\boldsymbol{P}$ & $\rho$ & $\boldsymbol{P}$ & $\rho$ & $\boldsymbol{P}$ & $\rho$ & $\boldsymbol{P}$ \\
\hline cFGF23 & -0.11 & 0.16 & -0.15 & 0.13 & -0.03 & 0.69 & -0.09 & 0.39 \\
\hline iFGF23 & -0.57 & $<0.001$ & -0.78 & $<0.001$ & -0.33 & $<0.001$ & -0.55 & $<0.001$ \\
\hline $1,25 D$ & 0.56 & $<0.001$ & 0.62 & $<0.001$ & 0.34 & $<0.001$ & 0.43 & $<0.001$ \\
\hline
\end{tabular}

1,25D, 1,25-dihydroxyvitamin D; CFGF23, C-terminal fibroblast growth factor 23; CKD, chronic kidney disease; FEPi, fractional excretion of phosphate; iFGF23 intact fibroblast growth factor 23; PTH, parathyroid hormone.

circulating concentration of iFGF23 rose more than 2-fold only in the ferric carboxymaltose group, and remained approximately 2-fold higher than baseline by week 1 before gradually decreasing towards baseline by week 5 . In contrast, we administered ferric carboxymaltose in 2 split doses of $750 \mathrm{mg}$ on days 0 and 7 , enrolled a larger population of patients with iron deficiency of more diverse causes, and did not collect data on day 1. Nevertheless, we observed an approximate 2-fold elevation of iFGF23 over baseline by week 1 (Table 3), which was similar to the week 1 change in the previous trial. By week 2, which was 1 week after the second 750-mg dose of ferric carboxymaltose was administered, we observed an additional near doubling of iFGF23 levels. Similarly, the approximate 50\% increase in PTH that we observed at week 5 exclusively in the ferric carboxymaltose-treated patients in the current study was similar to the effects of ferric carboxymaltose on PTH in the previous study. In summary, although the studies differed somewhat in their intravenous iron dosing schedules, timing of laboratory testing, and the composition of their study populations, the effects of ferric carboxymaltose on the phosphaturic hormones and on serum phosphate are reproducible findings across each study.

We administered single courses of the FDA-approved regimens of ferric carboxymaltose and ferumoxytol in the current randomized trial to parallel and inform contemporary clinical practice. Although it might be tempting to contend that the higher dose of elemental iron administered to the ferric carboxymaltose group (1,500 mg) versus the ferumoxytol group $(1,020 \mathrm{mg})$ accounted for its differential effects on phosphate homeostasis, iFGF23 was significantly elevated and hypophosphatemia had already developed in more than $20 \%$ of patients by week 1 after they had only received $750 \mathrm{mg}$ of ferric carboxymaltose, whereas only $1 \%$ of patients developed hypophosphatemia at any time after receiving 1,020 mg of elemental iron as ferumoxytol. When considered alongside the previous pilot trial that compared identical elemental iron doses of ferric carboxymaltose and iron dextran and found elevated iFGF23 and hypophosphatemia only in the ferric carboxymaltose group (15), our current results support the primacy of specific intravenous iron preparations in the pathogenesis of hypophosphatemia over differences in their doses.

Until now, no studies were large enough to systematically investigate risk factors for intravenous ironinduced hypophosphatemia. While ferric carboxymaltose exposure was the leading cause of incident hypophosphatemia and persistent hypophosphatemia at study end, other baseline factors influenced risk. Lower body weight increased risk, perhaps because the fixed dose of ferric carboxymaltose translated into relatively higher doses per body weight in patients with lower weight. This result suggests a dose-response effect within the ferric carboxymaltose group that we could not formally test given the fixed dosing. In addition, abnormal uterine bleeding relative to an unknown etiology of iron deficiency and Black versus White race were each associated with a nearly 2-fold higher risk of hypophosphatemia; higher baseline hemoglobin and lower serum phosphate and ferritin were also associated with increased risk. Notably, 25-hydroxyvitamin D insufficiency, which has been invoked as a potential facilitator of intravenous ironinduced hypophosphatemia, was not associated with risk of hypophosphatemia. While further studies are needed to investigate mechanisms of how these factors predispose to hypophosphatemia, clinicians should exercise special caution when prescribing ferric carboxymaltose to higher-risk patients. 
Hypophosphatemia was initially considered an uncommon, self-limited, and benign biochemical consequence of ferric carboxymaltose administration, but multiple cases with serious musculoskeletal complications have emerged (3-12). Across these cases, the correct diagnosis of hypophosphatemia was delayed by a confluence of factors including lack of knowledge of the relationship between iron and phosphate homeostasis; low awareness of hypophosphatemia as an adverse effect of ferric carboxymaltose; failure to test serum phosphate; and misattribution of symptoms of hypophosphatemia to iron deficiency anemia or its underlying cause even when serum phosphate was measured. Since most catastrophic cases occurred in individuals who received repeated courses of ferric carboxymaltose, we speculate that repeated administration of ferric carboxymaltose during the hypophosphatemic period caused by a previous dose induces a stacking effect that converts transient hypophosphatemia into protracted periods of severe hypophosphatemia. While our trial's design precluded us from assessing the clinical consequences of hypophosphatemia and how long they might persist after a single course of ferric carboxymaltose, a recent report describes severe respiratory symptoms following even one course (12). Clinicians should be aware of hypophosphatemia as a common adverse effect of ferric carboxymaltose administration and should consider strategies to mitigate the risk and consequences of hypophosphatemia, including standardizing serum phosphate testing, widening the interval of ferric carboxymaltose administration, or using alternative intravenous iron formulations.

\section{Methods}

Sample size and protocol. The FIRM trial (ClinicalTrials.gov, NCT02694978) recruited 1,997 patients according to its formal sample size calculation (24). For the nested physiological substudy, we anticipated that a sample size of approximately $10 \%$ of the overall study would be sufficient to support analyses of baseline clinical risk factors for incident hypophosphatemia. Based on needing approximately 10 patients per covariate to support stable multivariable models, and expecting approximately 18 covariates in the models, including 4 levels each for the categorical variables 'race' and 'etiology of iron deficiency anemia,' we recruited 185 patients.

We randomized patients 1:1 via a centralized Interactive Voice or web Response System (IXRS) to receive $510 \mathrm{mg}$ ferumoxytol or $750 \mathrm{mg}$ ferric carboxymaltose administered intravenously over $\geq 15$ minutes at baseline and 1 week later. We collected blood and urine samples at baseline (week 0) prior to randomization and at weeks 1 (prior to the second iron infusion), 2, and 5 after baseline. Although not required by protocol, virtually all study visits occurred in the mornings with blood draws performed in mid-morning.

Assays. We measured serum iron panels (iron, transferrin, and ferritin), hemoglobin, and serum and urine chemistries (creatinine, phosphate, and calcium) in all patients using standard clinical platforms at a central laboratory. In the nested physiological substudy, we also measured 25-hydroxyvitamin D, 1,25-dihydroxyvitamin D, and PTH using standard clinical assays, and FGF23 using cFGF23 and iFGF23 research assays from Immutopics.

Derived variables. We calculated TSAT as (iron/transferrin) $\times 100 ; \mathrm{FEPi}$ as (urine phosphate $\times$ serum creatinine $) /($ serum phosphate $\times$ urine creatinine $) \times 100$; and eGFR using the Modification of Diet in Renal Disease equation (32). We calculated within-patient percentage changes from baseline to week 1, 2, and 5 in the repeated measurements of phosphate homeostasis.

The reference laboratory reported serum phosphate concentrations in hundredths and considered values $<0.42 \mathrm{mmol} / 1(1.32 \mathrm{mg} / \mathrm{dl})$ as below the level of validation ( $n=123$ instances; $1.7 \%$ of all measurements during the trial). For analyses of serum phosphate and thus, FEPi as continuous variables, we conservatively assigned these a value of $0.41 \mathrm{mmol} / 1(1.27 \mathrm{mg} / \mathrm{dl})$. We defined hypophosphatemia as $<2.0 \mathrm{mg} / \mathrm{dl}$ in accordance with Common Terminology Criteria for Adverse Events, Grade 3, "severe" (33), and further subcategorized "extreme" hypophosphatemia as $<1.3 \mathrm{mg} / \mathrm{dl}$ (below the level of validation). While the initial FIRM trial report rounded serum phosphate to the nearest tenth and defined hypophosphatemia as $<0.6 \mathrm{mmol} / 1$ (24), here, we used the raw unrounded results in hundredths, which accurately rendered values of $0.60-0.64 \mathrm{mmol} / 1(1.86-1.98 \mathrm{mg} / \mathrm{dl})$ in the hypophosphatemia range of $<2.0 \mathrm{mg} / \mathrm{d} 1$.

Statistics. We used standard descriptive statistics to report baseline characteristics in the parent trial and the nested physiological substudy according to randomized group. Within the nested physiological substudy, we compared baseline concentrations of plasma cFGF23 and serum iFGF23 according to absence or presence of CKD, defined as eGFR $<60 \mathrm{ml} / \mathrm{min} / 1.73 \mathrm{~m}^{2}$, and used multiple linear regression with backward stepwise selection to identify parsimonious sets of independent determinants of each. 
We compared the overall incidence and visit-specific rates of severe and extreme hypophosphatemia ( $<2.0$ and $<1.3 \mathrm{mg} / \mathrm{dl}$ ) between treatment groups using Fisher's exact tests. We plotted visit-specific summary statistics of the repeated measurements of phosphate homeostasis in the nested physiological substudy. Depending on their distributions, we used 2-sample Student's $t$ tests or Wilcoxon's ranksum tests to compare between-group differences in absolute or percentage changes in the physiological parameters from baseline. We calculated Spearman's correlation coefficients to assess associations between changes in serum phosphate from baseline to week 2 and from baseline to week 5 relative to concomitant changes in the other physiological parameters in the nested substudy.

To investigate risk factors for incident hypophosphatemia and persistent hypophosphatemia at study end, we constructed multivariable logistic regression models that included randomized treatment group and baseline clinical factors as candidate predictors, and used backward stepwise selection to reach final parsimonious models.

Since moderate to advanced CKD impedes renal phosphate excretion (26), we stratified analyses of the parent trial by CKD status; due to the smaller sample size of the nested physiological substudy, we repeated its main analyses restricted to the subgroup of patients without CKD. All analyses were conducted using Stata/SE 14.2 (StataCorp). Statistical significance was inferred from 2-tailed $P$ values $<0.05$.

Study approval. This study was approved by the institutional review board at Quorum, 1501 Fourth Ave, Suite 800, Seattle, WA, USA, 98101. All patients provided written informed consent.

\section{Author contributions}

MW and WS designed the study, analyzed data, and drafted, edited, and approved the manuscript. GMC, ICM, RK, and JK designed the study and edited and approved the manuscript.

\section{Acknowledgments}

Sarah Greig, of inScience Communications, Springer Healthcare, provided editorial and referencing assistance, funded by AMAG Pharmaceuticals Inc.

Address correspondence to: Myles Wolf, MMSc, 2 Genome Court, Room 1009, Durham, North Carolina 27710, USA. Phone: 919.684.6752; Email: myles.wolf@duke.edu. Or to: William Strauss, AMAG Pharmaceuticals, Inc., 1100 Winter Street, Waltham, Massachusetts 02451, USA. Phone: 617.498.7789; Email: wstrauss@amagpharma.com.

1. Camaschella C. Iron-deficiency anemia. N Engl J Med. 2015;372(19):1832-1843

2. Lopez A, Cacoub P, Macdougall IC, Peyrin-Biroulet L. Iron deficiency anaemia. Lancet. 2016;387(10021):907-916.

3. Zoller H, Schaefer B, Glodny B. Iron-induced hypophosphatemia: an emerging complication. Curr Opin Nephrol Hypertens. 2017;26(4):266-275

4. Anand G, Schmid C. Severe hypophosphataemia after intravenous iron administration. BMJ Case Rep. 2017

5. Bartko J, Roschger P, Zandieh S, Brehm A, Zwerina J, Klaushofer K. Hypophosphatemia, severe bone pain, gait disturbance, and fatigue fractures after iron substitution in inflammatory bowel disease: a case report. J Bone Miner Res. 2018;33(3):534-539.

6. Klein K, Asaad S, Econs M, Rubin JE. Severe FGF23-based hypophosphataemic osteomalacia due to ferric carboxymaltose administration. BMJ Case Rep. 2018.

7. Schaefer B, Glodny B, Zoller H. Blood and bone loser. Gastroenterology. 2017;152(6):e5-e6.

8. Bishay RH, Ganda K, Seibel MJ. Long-term iron polymaltose infusions associated with hypophosphataemic osteomalacia: a report of two cases and review of the literature. Ther Adv Endocrinol Metab. 2017;8(1-2):14-19.

9. Reyes M, Diamond T. Hypophosphataemic rickets due to parenteral ferrous carboxymaltose in a young man with Crohn disease and iron deficiency: a case report and review of literature. J Clin Case Rep. 2017;7:931.

10. Edem D, Levitt H, Challinor SM. A case report of symptomatic hypophosphatemia secondary to elevated FGF-23 from parenteral ferric carboxymaltose treatment for iron deficiency anemia. Endocrine Society. https://www.endocrine.org/meetings/ endo-annual-meetings/abstract-details?ID=31602\&ID=31602. Published April 1, 2017. Accessed November 9, 2018.

11. Urbina T, et al. Iron supplementation-induced phosphaturic osteomalacia: FGF23 is the culprit. J Bone Miner Res. 2018;33(3):540-542.

12. Vasquez-Rios G, Marin E, Martin K, Merando A. Harder to breathe: an unusual case of severe hyperphosphaturic hypophosphatemia and normal FGF-23 levels in a young female patient. Am J Kid Dis. 2018;71:594.

13. Huang LL, et al. A controlled study of the effects of ferric carboxymaltose on bone and haematinic biomarkers in chronic kidney disease and pregnancy. Nephrol Dial Transplant. 2018;33(9):1628-1635.

14. Schouten BJ, Hunt PJ, Livesey JH, Frampton CM, Soule SG. FGF23 elevation and hypophosphatemia after intravenous iron polymaltose: a prospective study. J Clin Endocrinol Metab. 2009;94(7):2332-2337.

15. Wolf M, Koch TA, Bregman DB. Effects of iron deficiency anemia and its treatment on fibroblast growth factor 23 and phos- 
phate homeostasis in women. J Bone Miner Res. 2013;28(8):1793-1803.

16. Stöhr R, Sandstede L, Heine GH, Marx N, Brandenburg V. High-dose ferric carboxymaltose in patients with HFrEF induces significant hypophosphatemia. J Am Coll Cardiol. 2018;71(19):2270-2271.

17. Wolf M, White KE. Coupling fibroblast growth factor 23 production and cleavage: iron deficiency, rickets, and kidney disease. Curr Opin Nephrol Hypertens. 2014;23(4):411-419.

18. Jonsson KB, et al. Fibroblast growth factor 23 in oncogenic osteomalacia and X-linked hypophosphatemia. $N$ Engl J Med. 2003;348(17):1656-1663.

19. Shimada T, et al. Cloning and characterization of FGF23 as a causative factor of tumor-induced osteomalacia. Proc Natl Acad Sci USA. 2001;98(11):6500-6505.

20. ADHR Consortium. Autosomal dominant hypophosphataemic rickets is associated with mutations in FGF23. Nat Genet. 2000;26(3):345-348.

21. Schaefer B, et al. Choice of high-dose intravenous iron preparation determines hypophosphatemia risk. PLoS ONE. 2016;11(12):e0167146.

22. Bager P, Hvas CL, Dahlerup JF. Drug-specific hypophosphatemia and hypersensitivity reactions following different intravenous iron infusions. Br J Clin Pharmacol. 2017;83(5):1118-1125.

23. Hardy S, Vandemergel X. Intravenous iron administration and hypophosphatemia in clinical practice. Int J Rheumatol. 2015;2015:468675.

24. Adkinson NF, et al. Comparative safety of intravenous ferumoxytol versus ferric carboxymaltose in iron deficiency anemia: A randomized trial. Am J Hematol. 2018;93(5):683-690

25. Farrow EG, et al. Iron deficiency drives an autosomal dominant hypophosphatemic rickets (ADHR) phenotype in fibroblast growth factor-23 (Fgf23) knock-in mice. Proc Natl Acad Sci USA. 2011;108(46):E1146-E1155.

26. Wolf M. Update on fibroblast growth factor 23 in chronic kidney disease. Kidney Int. 2012;82(7):737-747

27. Larsson T, et al. Fibroblast growth factor-23 mutants causing familial tumoral calcinosis are differentially processed. Endocrinology. 2005;146(9):3883-3891.

28. Shimada T, et al. Circulating fibroblast growth factor 23 in patients with end-stage renal disease treated by peritoneal dialysis is intact and biologically active. J Clin Endocrinol Metab. 2010;95(2):578-585.

29. David V, et al. Inflammation and functional iron deficiency regulate fibroblast growth factor 23 production. Kidney Int. 2016;89(1):135-146

30. Hu MC, Shiizaki K, Kuro-o M, Moe OW. Fibroblast growth factor 23 and Klotho: physiology and pathophysiology of an endocrine network of mineral metabolism. Annu Rev Physiol. 2013;75:503-533.

31. Isakova T, et al. Fibroblast growth factor 23 is elevated before parathyroid hormone and phosphate in chronic kidney disease. Kidney Int. 2011;79(12):1370-1378.

32. Levey AS, et al. Using standardized serum creatinine values in the modification of diet in renal disease study equation for estimating glomerular filtration rate. Ann Intern Med. 2006;145(4):247-254.

33. Common Terminology Criteria for Adverse Events (CTCAE), version 4.0. US Department of Health Human Services. https://www.eortc.be/services/doc/ctc/CTCAE_4.03_2010-06-14_QuickReference_5x7.pdf. Published May 28, 2009. Accessed November 9, 2018. 